\title{
Article \\ On the Semi-Local Convergence of an Ostrowski-Type Method for Solving Equations
}

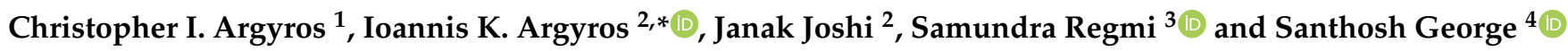 \\ 1 Department of Computing and Technology, Cameron University, Lawton, OK 73505, USA; \\ Christopher.Argyros@cameron.edu \\ 2 Department of Mathematical Sciences, Cameron University, Lawton, OK 73505, USA; jjoshi@cameron.edu \\ 3 Learning Commons, University of North Texas at Dallas, Dallas, TX 75201, USA; \\ samundra.regmi@untdallas.edu \\ 4 Department of Mathematical and Computational Sciences, National Institute of Technology Karnataka, \\ Mangalore 575025, India; sgeorge@nitk.edu.in \\ * Correspondence: iargyros@cameron.edu
}

check for

updates

Citation: Argyros, C.I.; Argyros, I.K.; Joshi, J.; Regmi, S.; George, S. On the Semi-Local Convergence of an Ostrowski-Type Method for Solving Equations. Symmetry 2021, 13, 2281. https://doi.org/10.3390/sym13122281

Academic Editor: Dongfang Li

Received: 13 October 2021

Accepted: 18 November 2021

Published: 1 December 2021

Publisher's Note: MDPI stays neutral with regard to jurisdictional claims in published maps and institutional affiliations.

Copyright: (c) 2021 by the authors. Licensee MDPI, Basel, Switzerland. This article is an open access article distributed under the terms and conditions of the Creative Commons Attribution (CC BY) license (https:// creativecommons.org/licenses/by/ $4.0 /)$.

\begin{abstract}
Symmetries play a crucial role in the dynamics of physical systems. As an example, microworld and quantum physics problems are modeled on principles of symmetry. These problems are then formulated as equations defined on suitable abstract spaces. Then, these equations can be solved using iterative methods. In this article, an Ostrowski-type method for solving equations in Banach space is extended. This is achieved by finding a stricter set than before containing the iterates. The convergence analysis becomes finer. Due to the general nature of our technique, it can be utilized to enlarge the utilization of other methods. Examples finish the paper.
\end{abstract}

Keywords: Ostrowski-type method; Banach space; convergence criterion

\section{Introduction}

We are concerned with finding $x_{*}$ solving

$$
F(x)=0,
$$

where $F: D \subset E \longrightarrow E_{1}$ is an operator acting between Banach spaces $E$ and $E_{1}$ with $D \neq \varnothing$.

The famous Ostrowski-type method is defined for $x_{0} \in D$ and each $n=0,1,2, \ldots$ by

$$
\begin{aligned}
y_{k} & =x_{k}-F^{\prime}\left(x_{k}\right)^{-1} F\left(x_{k}\right) \\
x_{k+1} & =y_{k}-A_{k} F\left(x_{k}\right),
\end{aligned}
$$

where $A_{k}=2\left[y_{k}, x_{k} ; F\right]^{-1}-F^{\prime}\left(x_{k}\right)^{-1}$, with $[., ., F]: D \times D \rightarrow L\left(E, E_{1}\right)$. There are numerous results for the convergence of iterative methods utilizing the information $\left(D, x_{0}, F, F^{\prime}\right)$ and higher order derivatives [1-39]. However, higher order derivatives cannot be found on method (2). Moreover, these results do not give uniqueness ball or estimates on $\left\|x_{k}-x_{*}\right\|$ or $\left\|x_{k+1}-x_{k}\right\|$. That is why we are motivated to write this paper, where only hypotheses on the derivative and divided differences of order one are used. Notice that only these operators appear on method (2).

The method (2) is shown to be of order four using Taylor expansion and assumptions on the fifth order derivative of $F$, which is not on these schemes [5]. So, the assumptions on the sixth derivative reduce the applicability of this method.

For example: Let $E=E_{1}=\mathbb{R}, D=[-0.5,1.5]$. Define $\lambda$ on $D$ by

$$
\lambda(t)=\left\{\begin{array}{cc}
t^{3} \log t^{2}+t^{5}-t^{4} & \text { if } t \neq 0 \\
0 & \text { if } t=0 .
\end{array}\right.
$$


Then, we get $t^{*}=1$, and

$$
\lambda^{\prime \prime \prime}(t)=6 \log t^{2}+60 t^{2}-24 t+22
$$

Obviously, $\lambda^{\prime \prime \prime}(t)$ is not bounded on $D$. So, the convergence of method (2) is not guaranteed by the previous analyses in [5].

The rest of the study is organized as follows: Section 2 contains results on majorizing sequences. In Section 3, we develop the semi-local convergence analysis based on majorizing sequences. The local convergence analysis can be found in Section 4 . Numerical examples can be found in Section 5. The paper ends with some concluding remarks in Section 6.

\section{Majorizing Sequences}

We recall the definition of a majorizing sequences.

Definition 1. Let $\left\{v_{k}\right\}$ be a sequence in a complete normed space. Then, a non-decreasing scalar sequence $\left\{d_{k}\right\}$ is called majorizing for $\left\{v_{k}\right\}$ if

$$
\left\|v_{k+1}-v_{k}\right\| \leq d_{k+1}-d_{k} \text { for each } k=0,1,2, \ldots
$$

Then, the convergence of sequence $\left\{v_{k}\right\}$ reduces to studying that of $\left\{d_{k}\right\}$ [40].

Let $\eta \geq 0$ and $L, L_{i}, i=0,1,2,3,4$ be positive parameters. Set $M_{0}=\frac{L_{0} L_{2}}{2}$, $M=\frac{L}{2}, M_{1}=\frac{L L_{2}}{2}$ and $M_{2}=\frac{L L_{3}}{2}$. Define sequences $\left\{t_{k}\right\},\left\{s_{k}\right\},\left\{\alpha_{k}\right\}$ and $\left\{\beta_{k}\right\}$ for each $k=0,1,2, \ldots$ by $t_{0}=0, s_{0}=\eta$

$$
\begin{aligned}
t_{1}= & s_{0}+\frac{M_{0} s_{0}^{2}}{1-L_{1} s_{0}}+\frac{M_{2} s_{0}^{3}}{\left(1-L_{1} s_{0}\right)\left(1-L_{0} s_{0}\right)}+\frac{M s_{0}^{2}}{1-L_{0} s_{0}}, \\
s_{1}= & t_{1}+\frac{M\left(t_{1}-t_{0}\right)^{2}+t_{1}-s_{0}}{1-L_{0} t_{1}} \\
t_{k+1}= & s_{k}+\alpha_{k}\left(s_{k}-t_{k}\right), \\
s_{n+1}= & t_{n+1}+\frac{M\left(t_{n+1}-t_{n}\right)^{2}+L_{4} t_{n}\left(t_{n+1}-s_{n}\right)}{1-L_{0} t_{n+1}}, \\
\alpha_{k}= & \frac{M_{1}\left(s_{k}-t_{k}\right)}{\left(1-L_{1}\left(s_{k}+t_{k}\right)\right)\left(1-L_{0} t_{k}\right)} \\
& +\frac{M_{2}\left(s_{k}-t_{k}\right)}{\left(1-L_{1}\left(s_{k}+t+k\right)\right)\left(1-L_{0} s_{k}\right)}+\frac{M\left(s_{k}-t_{k}\right)}{1-L_{0} s_{k}}, \\
\beta_{k}= & \frac{M\left(t_{k+1}-t_{k}\right)+L_{4} t_{k}}{1-L_{0} t_{k+1}} .
\end{aligned}
$$

Moreover, define quadratic polynomials and functions on the interval $[0,1]$ for some $b>1$

$$
\begin{gathered}
p_{1}(t)=t^{2}-\left(1-L_{0} t_{1}\right) t+L_{4} t_{1} \\
p_{2}(t)=t^{2}+t-\left(1-\frac{2 b L_{1} t_{1}}{b-1}\right), \\
p_{3}(t)=t^{2}+t-\left(1-L_{0} t_{1}\right), \\
g_{1}(t)=\left(M_{1} b+M_{2} b+M\right) t
\end{gathered}
$$

and

$$
\left.g_{2}(t)=M t((1+t) t-1)(1+t)\right)+(1+t)^{2} t^{2}\left(L_{4}+L_{0} t^{2}(1+t)\right) .
$$


Denote by $\gamma_{0}, \gamma_{1}$ or $\gamma_{2}$ or $\gamma_{3}, \gamma_{4}$, the non-negative zeros of $p_{1}, p_{2}, p_{3}$ if they exist. Furthermore, define sequences of functions on the interval $[0,1]$ for $\delta=\delta(t)=(1+t) t$ by

$$
f_{n}^{(1)}(t)=M_{1} b t \delta^{n-1} t_{1}+M_{2} b t \delta^{n-1} t_{1}+M t \delta^{n-1} t_{1}+L_{0} t\left(1+\delta+\ldots+\delta^{n}\right) t_{1}-t
$$

and

$$
f_{n}^{(2)}(t)=M\left(t^{2} \delta^{n-1}+t \delta^{n-1}\right) t_{1}+L_{4}\left(1+\delta+\ldots+\delta^{n}\right) t_{1}+L_{0} t\left(1+\delta+\ldots+\delta^{n}\right) t_{1}-t .
$$

Next, we present two results on the majorizing sequence for method (2).

Lemma 1. Suppose that for each $k=0,1,2, \ldots$, items

$$
s_{k} \leq t_{k+1}<\frac{1}{L_{0}}
$$

and

$$
s_{k}+t_{k}<\frac{1}{L_{1}}
$$

hold. Then, sequences $\left\{s_{k}\right\}$ and $\left\{t_{k}\right\}$ are increasing, bounded from above by $\frac{1}{L_{0}}$ and converge to their unique least upper bound $s_{*} \in\left[0, \frac{1}{L_{0}}\right]$.

Proof. It follows from (3)-(5) that sequences $\left\{s_{k}\right\},\left\{t_{k}\right\}$ are increasing, bounded from above by $\frac{1}{L_{0}}$ and as such they converge to $s_{*}$.

Remark 1. Conditions (4) and (5) hold only in some special cases. This is why we present stronger conditions that can be verified more easily.

We shall use the following set of conditions denoted by (A) in our second result on majorizing sequences for method (2).

Suppose: there exists $\gamma \in S:=\left(0, \frac{\sqrt{5}-1}{2}\right), b>0, \eta>0$ satisfying

$$
\begin{aligned}
0 & \leq \alpha_{0} \leq \gamma, 0 \leq \beta_{0} \leq \gamma, \\
L_{0} \eta & <1, L_{1} \eta<1, L_{1} t_{1}<\frac{b-1}{2 b}, \\
\gamma_{0} & \leq \gamma \leq \gamma_{1} \text { if } g_{2}(t) \geq 0 \text { for each } t \in S,
\end{aligned}
$$

or

$$
\begin{aligned}
f_{1}^{(2)}(\gamma) & \leq 0 \text { if } g_{2}(t) \leq 0 \text { for each } t \in S \\
\gamma & \leq \gamma_{2} \\
\text { and } & \\
\gamma_{3} & \leq \gamma \leq \gamma_{4} .
\end{aligned}
$$

Then, under the preceding notation and conditions (A), we can show.

Lemma 2. Under conditions (A), the conclusions of Lemma 1 hold for sequences $\left\{s_{k}\right\},\left\{t_{k}\right\}$. Moreover, the following assertions hold for each $k=0,1,2, \ldots$

$$
\begin{gathered}
0 \leq s_{k}-t_{k} \leq \gamma^{k}(1+\gamma)^{k-1}\left(t_{1}-t_{0}\right) \\
0 \leq t_{k+1}-s_{k} \leq \gamma^{k+1}(1+\gamma)^{k-1}\left(t_{1}-t_{0}\right), \\
0 \leq s_{k} \leq \frac{1-\delta^{k+1}}{1-\delta} t_{1}
\end{gathered}
$$


and

$$
0 \leq t_{k+1} \leq \frac{1-\delta^{n+2}}{1-\delta} t_{1}
$$

Recall that $\delta=\gamma(\gamma+1)$ and $\delta(t)=\delta$.

Proof. We shall show using induction on $n$ that the following hold.

$$
\begin{gathered}
0 \leq \alpha_{n} \leq \gamma, \\
0 \leq \beta_{n} \leq \gamma, \\
L_{0} t_{n} \leq 1, L_{0} s_{n}<1, L_{1}\left(s_{n}+t_{n}\right)<1,
\end{gathered}
$$

and

$$
t_{n} \leq s_{n} \leq t_{n+1} .
$$

Estimates (10)-(13) hold for $n=0$, by the initial conditions and conditions (A). We also have

$$
\begin{aligned}
0 \leq & s_{0}-t_{0} \leq \eta, 0 \leq t_{1}-s_{0} \leq \gamma \eta, 0 \leq s_{1}-t_{1} \leq \gamma\left(t_{1}-t_{0}\right) \\
0 \leq & t_{2}-s_{1} \leq \gamma^{2}\left(t_{1}-t_{0}\right) \\
0 \leq & s_{2}-t_{2} \leq \gamma^{2}(1+\gamma)\left(t_{1}-t_{0}\right) \\
0 \leq & t_{3}-s_{2} \leq \gamma^{3}(1+\gamma)\left(t_{1}-t_{0}\right), \\
& \vdots \\
0 \leq & s_{n}-t_{n} \leq \gamma^{n}(1+\gamma)^{n-1}\left(t_{1}-t_{0}\right), \\
0 \leq & t_{n+1}-s_{n} \leq \gamma^{n+1}(1+\gamma)^{n-1}\left(t_{1}-t_{0}\right), \\
t_{n+1} \leq & s_{n}+\gamma^{n+1}(1+\gamma)^{n-1}\left(t_{1}-t_{0}\right) \leq t_{n}+\gamma^{n}(1+\gamma)^{n-1}\left(t_{1}-t_{0}\right) \\
& +\gamma^{n+1}(1+\gamma)^{n-1}\left(t_{1}-t_{0}\right) \\
& \vdots \quad \\
\leq & t_{1}+\gamma(1+\gamma)^{n-1}\left(t_{1}-t_{0}\right)+\gamma^{2}(1+\gamma)\left(t_{1}-t_{0}\right)+ \\
\vdots & \\
& +\gamma^{n}(1+\gamma)^{n-1}\left(t_{1}-t_{0}\right)+\gamma^{n+1}(1+\gamma)^{n-1}\left(t_{1}-t_{0}\right) \\
\leq & \left(1+\delta+\ldots+\delta^{n+1}\right) t_{1}=\frac{1-\delta^{n+2}}{1-\delta} t_{1}
\end{aligned}
$$

and

$$
s_{n} \leq t_{n}+\gamma^{n}(1+\gamma)^{n-1} t_{1} \leq \ldots \leq \frac{1-\delta^{n+1}}{1-\delta} t_{1} .
$$

Suppose these estimates hold for all integers smaller or equal to $n$. Then, evidently, (10) holds (since $\frac{1}{1-L_{1}\left(s_{n}+t_{n}\right)} \leq b$ ), if we show instead using (14)-(17) that

$$
\frac{M_{1} b\left(s_{n}-t_{n}\right)}{1-L_{0} t_{n}}+\frac{M_{2} b\left(s_{n}-t_{n}\right)}{1-L_{0} s_{n}}+\frac{M\left(s_{n}-t_{n}\right)}{1-L_{0} s_{n}} \leq \gamma
$$

or

$$
\begin{aligned}
& M_{1} b \gamma \delta^{n-1} t_{1}+M_{2} b \gamma \delta^{n-1} t_{1}+M \gamma \delta^{n-1} t_{1} \\
& +\gamma L_{0}\left(1+\delta+\ldots+\delta^{n}\right) t_{1}-\gamma \leq 0 .
\end{aligned}
$$

Notice that expression (19) is obtained if we replace $s_{n}-t_{n}, t_{n}, s_{n}$ by the right hand sides of (14), (15) and (17), respectively, in (18), remove denominators and move all terms at the right hand side of the inequality. 
Estimate (19) motivates us to define functions $f_{n}^{(1)}$ on the interval $[0,1]$ and show instead of (19)

$$
f_{n}^{(1)}(t) \leq 0 \text { at } t=\gamma .
$$

We shall find a relationship between two consecutive functions $f_{n}^{(1)}$. We can write in turn that

$$
\begin{aligned}
f_{n+1}^{(1)}(t)= & M_{1} b t \delta^{n} t_{1}+M_{2} b t \delta^{n} t_{1}+M t \delta^{n} t_{1}+L_{0} t\left(1+\delta+\ldots+\delta^{n+1}\right) t_{1}-t \\
& -M_{1} b t \delta^{n-1} t_{1}-M_{2} b t \delta^{n-1} t_{1}-M t \delta^{n-1} t_{1} \\
& -L_{0} t\left(1+\delta+\ldots+\delta^{n}\right) t_{1}+t+f_{n}^{(1)}(t) \\
= & f_{n}^{(1)}(t)+\left(M_{1} b t \delta^{n} t_{1}-M_{1} b t \delta^{n-1} t_{1}\right) \\
& +\left(M_{2} b t \delta^{n} t_{1}-M_{2} b t \delta^{n-1} t_{1}\right)+\left(M t \delta^{n} t_{1}-M t \delta^{n-1} t_{1}\right)+t L_{0} \delta^{n+1} t_{1} \\
= & f_{n}^{(1)}(t)+(\delta-1) t\left(M_{1} b+M_{2} b+M\right) \delta^{n-1} t_{1} \\
= & f_{n}^{(1)}(t)+(\delta-1) g_{1}(t) \delta^{n-1} t_{1} \\
\leq & f_{n}^{(1)}(t),
\end{aligned}
$$

since $t \in\left[0, \frac{\sqrt{5}-1}{2}\right]$, so

$$
f_{n+1}^{(1)}(t) \leq f_{n}^{(1)}(t)
$$

Define function

$$
f_{\infty}^{(1)}(t)=\lim _{k \longrightarrow+\infty} f_{k}^{(1)}(t) .
$$

By the definition of functions $f_{n}^{(1)}$ and $f_{\infty}^{(1)}$, we get

$$
f_{\infty}^{(1)}(t)=\frac{t L_{0} t_{1}}{1-\delta}-t .
$$

Then, we can show instead of (20) that

$$
f_{\infty}^{(1)}(t) \leq 0 \text { at } t=\gamma,
$$

which is true by the definition of $p_{3}$ and $\gamma_{3} \leq \gamma \leq \gamma_{4}$. Similarly, (11) holds if

$$
\begin{aligned}
& M\left(\gamma^{2} \delta^{n-1}+\gamma \delta^{n-1}\right) t_{1}+L_{4}\left(1+\delta+\ldots+\delta^{n}\right) t_{1} \\
& +L_{0} \gamma\left(1+\delta+\ldots+\delta^{n+1}\right) t_{1}-\gamma \leq 0
\end{aligned}
$$

or

$$
f_{n}^{(2)}(t) \leq 0 \text { at } t=\gamma
$$

This time, we have

$$
\begin{aligned}
f_{n+1}^{(2)}(t)= & M\left(t^{2} \delta^{n}+t \delta^{n}\right) t_{1}+L_{4}\left(1+\delta+\ldots+\delta^{n+1}\right) t_{1} \\
& +L_{0} t\left(\left(1+\delta+\ldots+\delta^{n+2}\right) t_{1}-t\right. \\
& -M\left(t^{2} \delta^{n-1}+t \delta^{n-1}\right) t_{1}-L_{4}\left(1+\delta+\ldots+\delta^{n}\right) t_{1} \\
& -L_{0} t\left(1+\delta+\ldots+\delta^{n+1}\right) t_{1}+t+f_{n}^{(2)}(t) \\
= & f_{n}^{(2)}+M\left(t^{2} \delta^{n}+t \delta^{n}-t^{2} \delta^{n-1}-t \delta^{n-1}\right) t_{1} \\
& +L_{4} \delta^{n+1} t_{1}+L_{0} t \delta^{n+2} t_{1} \\
= & f_{n}^{(2)}(t)+g_{2}(t) \delta^{n-1} t_{1},
\end{aligned}
$$




$$
f_{n+1}^{(2)}(t)=f_{n}^{(2)}(t)+g_{2}(t) \delta^{n-1} t_{1}
$$

Define function

$$
f_{\infty}^{(2)}(t)=\lim _{k \longrightarrow+\infty} f_{n}^{(2)}(t) .
$$

Then, we get

$$
f_{\infty}^{(2)}(t)=\frac{L_{4} t_{1}}{1-t}+\frac{L_{0} t t_{1}}{1-t}-t .
$$

If $\gamma_{0} \leq \gamma \leq \gamma_{1}$, then $g_{2}(t) \geq 0$ for each $t \in S$ and $f_{\infty}^{(2)}(t) \leq 0$ holds at $t=\gamma$. However, if $g_{2}(t) \leq 0$ for each $t \in S$, then

$$
f_{n+1}^{(2)}(t) \leq f_{n}^{(2)}(t) .
$$

In this case, (26) holds if $f_{1}^{(2)}(t) \leq 0$ at $t=\gamma$, which is true. Therefore, the induction for (10)-(13) is completed. Hence, sequences $\left\{s_{k}\right\},\left\{t_{k}\right\}$ are non-decreasing, bounded from above by $\frac{t_{1}}{1-\delta} t_{1}$ and as such they converge to $s_{*}$ satisfying $s_{*} \in\left[\eta, \frac{t_{1}}{1-\delta}\right]$.

\section{Semi-Local Convergence}

We shall use conditions $(\mathrm{H})$ :

Suppose

(H1) There exist $x_{0} \in D, \eta \geq 0$ such that $F^{\prime}\left(x_{0}\right)$ is invertible and

$$
\left\|F^{\prime}\left(x_{0}\right)^{-1} F\left(x_{0}\right)\right\| \leq \eta .
$$

(H2) For each $u \in D$

$$
\left\|F^{\prime}\left(x_{0}\right)^{-1}\left(F^{\prime}(u)-F^{\prime}\left(x_{0}\right)\right)\right\| \leq L_{0}\left\|u-x_{0}\right\| .
$$

Set $D_{0}=U\left(x_{*}, \frac{1}{L_{0}}\right) \cap D$.

(H3) For each $v, w \in D_{0}$

$$
\begin{gathered}
\left\|F^{\prime}\left(x_{0}\right)^{-1}\left(F^{\prime}(w)-F^{\prime}(v)\right)\right\| \leq L\|w-v\|, \\
\left\|F^{\prime}\left(x_{0} *\right)^{-1}\left([v, w ; F]-F^{\prime}\left(x_{0}\right)\right)\right\| \leq L_{1}\left(\left\|v-x_{0}\right\|+\left\|w-x_{0}\right\|\right), \\
\left\|F^{\prime}\left(x_{0}\right)^{-1}\left([v, w ; F]-F^{\prime}(w)\right)\right\| \leq L_{2}\|v-w\| \\
\left\|F^{\prime}\left(x_{0}\right)^{-1}\left([v, w ; F]-F^{\prime}(v)\right)\right\| \leq L_{3}\|v-w\| .
\end{gathered}
$$

and

$$
\left\|F^{\prime}\left(x_{0}\right)^{-1} F^{\prime}(w)\right\| \leq L_{4}\left\|w-x_{0}\right\| .
$$

(H4) $U\left[x_{0}, s_{*}\right] \subset D$

$$
\text { and }
$$

(H5) Conditions of Lemma 1 or Lemma 2 hold.

Then, based on conditions $(\mathrm{H})$, we present the semi-local convergence analysis of method (2).

Theorem 1. Suppose hypotheses (H) hold. Then, sequences $\left\{x_{k}\right\},\left\{y_{k}\right\}$ generated by method (2) with starter $x_{0}$ are well defined in $U\left[x_{0}, s_{*}\right]$, remain in $U\left[x_{0}, s_{*}\right]$ for each $n=0,1,2, \ldots$ and converge to a solution $x_{*} \in U\left[x_{0}, s_{*}\right]$ of equation $F(x)=0$. Moreover, the following error estimates hold

$$
\left\|x_{k}-x_{*}\right\| \leq t_{n}-s_{*} .
$$

Proof. Mathematical induction is employed to show

$$
\left\|x_{k+1}-y_{k}\right\| \leq t_{k+1}-s_{k}
$$


and

$$
\left\|y_{k}-x_{k}\right\| \leq s_{k}-t_{k} .
$$

Iterate $y_{0}$ is well defined by the first substep of method (2) and (H1). We can write

$$
\left\|y_{0}-x_{0}\right\|=\left\|F^{\prime}\left(x_{0}\right)^{-1} F\left(x_{0}\right)\right\| \leq \eta=s_{0}-t_{0}=s_{0} \leq s_{*},
$$

so $y_{0} \in U\left[x_{0}, s_{*}\right]$. Using $(\mathrm{H} 3)$, we get in turn for $v, w \in U\left(x_{0}, s_{*}\right)$

$$
\begin{aligned}
\left\|F^{\prime}\left(x_{0}\right)^{-1}\left([v, w ; F]-F^{\prime}\left(x_{0}\right)\right)\right\| & \leq L_{1}\left(\left\|v-x_{0}\right\|+\left\|w-x_{0}\right\|\right) \\
& \leq L_{1}\left(s_{*}+s_{*}\right)=2 L_{1} s_{*}<1
\end{aligned}
$$

by the Lemma on invertible opertors due to Banach [41,42], leading to

$$
\left\|[v, w ; F]^{-1} F^{\prime}\left(x_{0}\right)\right\| \leq \frac{1}{1-L_{1}\left(\left\|v-x_{0}\right\|+\left\|w-x_{0}\right\|\right)} .
$$

Similarly, iterate $x_{1}$ is well defined by the second substep of method (2). We also have by (H2) for $w \in U\left(x_{0}, s^{*}\right)$

$$
\left\|F^{\prime}\left(x_{0}\right)^{-1}\left(F^{\prime}(w)-F^{\prime}\left(x_{0}\right)\right)\right\| \leq L_{0}\left\|w-x_{0}\right\| \leq L_{0} s^{*}<1,
$$

so $F^{\prime}(w)^{-1} \in L\left(E_{1}, E\right)$ and

$$
\left\|F^{\prime}(w)^{-1} F^{\prime}\left(x_{0}\right)\right\| \leq \frac{1}{1-L_{0}\left\|w-x_{0}\right\|} .
$$

Hence, by (35) for $v=y_{0}$ and $w=x_{0}$ and (36) for $w=x_{0}$, we have

$$
\begin{aligned}
x_{1}-y_{0}= & -\left[y_{0}, x_{0} ; F\right]^{-1}\left(F^{\prime}\left(x_{0}\right)-\left[y_{0}, x_{0} ; F\right]\right) F^{\prime}\left(x_{0}\right)^{-1} F\left(y_{0}\right) \\
& -\left[y_{0}, x_{0} ; F\right]^{-1}\left(F^{\prime}\left(y_{0}\right)-\left[y_{0}, x_{0} ; F\right]\right) F^{\prime}\left(y_{0}\right)^{-1} F\left(y_{0}\right) \\
& -F^{\prime}\left(y_{0}\right)^{-1} F\left(y_{0}\right) .
\end{aligned}
$$

In view of (H3), (35), (36) (for $\left.v=y_{0}, w=x_{0}\right)$, (37) and triangle inequality, we get in turn

$$
\begin{aligned}
\left\|x_{1}-y_{0}\right\| \leq & \frac{L L_{2}\left\|y_{0}-x_{0}\right\|\left\|y_{0}-x_{0}\right\|^{2}}{2\left(1-L_{1}\left(\left\|y_{0}-x_{0}\right\|+\left\|x_{0}-x_{0}\right\|\right)\right)\left(1-L_{0}\left\|x_{0}-x_{0}\right\|\right)} \\
& +\frac{L L_{3}\left\|y_{0}-x_{0}\right\|\left\|y_{0}-x_{0}\right\|^{2}}{2\left(1-L_{1}\left(\left\|y_{0}-x_{0}\right\|+\left\|x_{0}-x_{0}\right\|\right)\right)\left(1-L_{0}\left\|y_{0}-x_{0}\right\|\right)} \\
& +\frac{L\left\|y_{0}-x_{0}\right\|^{2}}{2\left(1-L_{0}\left\|y_{0}-x_{0}\right\|\right)} \leq \alpha_{0}\left(s_{0}-t_{0}\right)=t_{1}-s_{0}
\end{aligned}
$$

and

$$
\left\|x_{1}-x_{0}\right\| \leq\left\|x_{1}-y_{0}\right\|+\left\|y_{0}-x_{0}\right\| \leq t_{1}-s_{0}+s_{0}-t_{0}=t_{1} \leq s_{*},
$$

so $x_{1} \in U\left[x_{0}, s_{*}\right]$. Thus, estimates (32) and (33) hold for $n=0$, where we also used

$$
\begin{aligned}
\left\|F^{\prime}\left(x_{0}\right)^{-1} F\left(y_{0}\right)\right\| & =\left\|\int_{0}^{1} F^{\prime}\left(x_{0}\right)^{-1}\left(F^{\prime}\left(x_{0}+\theta\left(y_{0}-x_{0}\right)\right)-F^{\prime}\left(x_{0}\right)\right)\left(y_{0}-x_{0}\right) d \theta\right\| \\
& \leq \frac{L_{0}}{2}\left\|y_{0}-x_{0}\right\|^{2} \leq \frac{L}{2}\left(s_{0}-t_{0}\right)^{2} .
\end{aligned}
$$


We know that (36) holds for $w=x_{1}$, so iterate $y_{1}$ is well defined by the first substep of method (2) for $n=1$, and we can write

$$
\begin{aligned}
F\left(x_{1}\right)= & F\left(x_{1}\right)-F\left(x_{0}\right)-F^{\prime}\left(x_{0}\right)\left(x_{1}-x_{0}\right) \\
& +F^{\prime}\left(x_{0}\right)\left(x_{1}-y_{0}\right) \\
= & \int_{0}^{1}\left(F^{\prime}\left(x_{0}+\theta\left(x_{1}-x_{0}\right)\right)-F^{\prime}\left(x_{0}\right)\right)\left(x_{1}-x_{0}\right) d \theta+F^{\prime}\left(x_{0}\right)\left(x_{1}-y_{0}\right) .
\end{aligned}
$$

Then, we obtain by method (2), (36) (for $\left.w=x_{1}\right),(40)$ and the triangle inequality

$$
\begin{aligned}
\left\|y_{1}-x_{1}\right\| & \leq \frac{\frac{L}{2}\left\|x_{1}-x_{0}\right\|^{2}+\left\|x_{1}-y_{0}\right\|}{1-L_{0}\left\|x_{1}-x_{0}\right\|} \\
& \leq \frac{M\left(t_{1}-t_{0}\right)^{2}+t_{1}-s_{0}}{1-L_{0} t_{1}}=s_{1}-t_{1} .
\end{aligned}
$$

Then, we have

$$
\left\|y_{1}-x_{0}\right\| \leq\left\|y_{1}-x_{1}\right\|+\left\|x_{1}-x_{0}\right\| \leq s_{1}-t_{1}+t_{1}=s_{1} \leq s_{*},
$$

so $y_{1} \in U\left[x_{0}, s_{*}\right]$. Suppose estimates (32) and (33) hold for all integers smaller or equal to $n-1$. Then, simply repeat the preceding calculations with $x_{0}, y_{0}, x_{1}$ replaced by $x_{m}, y_{m}, x_{m+1}$, respectively, and use the induction hypotheses to terminate the proof for (32) and (33). By the Lemma sequence $\left\{t_{k}\right\}$ is Cauchy in a Banach space $E$ and as such it converges to some $x_{*} \in U\left[x_{0}, s_{*}\right]$ since it is a closed set. Finally, using (40), we get

$$
\begin{aligned}
\left\|F^{\prime}\left(x_{0}\right)^{-1} F\left(x_{k+1}\right)\right\| \leq & \frac{L}{2}\left\|x_{k+1}-x_{k}\right\|^{2} \\
& +L_{4}\left\|x_{k}-x_{0}\right\|\left\|x_{k+1}-x_{k}\right\| \\
\leq & \frac{L}{2}\left(t_{k+1}-t_{k}\right)^{2}+L_{4} t_{k}\left(t_{k+1}-s_{k}\right) \longrightarrow 0
\end{aligned}
$$

as $n \longrightarrow+\infty$ implying $F\left(x_{*}\right)=0$ (by the continuity of $F$ ).

The point $s_{*}$ can be replaced by $\frac{1}{L_{0}}$ or $\frac{t_{1}}{1-\delta}$, respectively, given in closed form.

Next, a uniqueness of the solution $x_{*}$ of equation $F(x)=0$ is presented.

Proposition 1. Suppose:

(a) There exists a solution $x_{*} \in D$ of equation $F(x)=0$;

(b) There exists $s \geq s_{*}$ such that

$$
\frac{L_{0}}{2}\left(s+s_{*}\right)<1 .
$$

Set $D_{1}=U\left[x_{0}, s_{*}\right] \cap D$. Then, the only solution of equation $F(x)=0$ in the region $D_{1}$ is $x_{*}$.

Proof. Let $x^{* *} \in D_{1}$ with $F\left(x^{* *}\right)=0$. Set $M=\int_{0}^{1} F^{\prime}\left(x^{* *}+\theta\left(x_{*}-x^{* *}\right)\right) d \theta$. Using (H2) and (42), we obtain in turn that

$$
\begin{aligned}
\left\|F^{\prime}\left(x_{0}\right)^{-1}\left(M-F^{\prime}\left(x_{0}\right)\right)\right\| & \leq L_{0} \int_{0}^{1}\left((1-\theta)\left\|x_{*}-x_{0}\right\|+\theta\left\|x^{* *}-x_{0}\right\|\right) d \theta \\
& \leq L_{0} \int_{0}^{1}(1-\theta) s d \theta+L_{0} \int_{0}^{1} \theta s_{*} d \theta \\
& \leq \frac{L_{0}}{2}\left(s+s_{*}\right)<1
\end{aligned}
$$

so $x^{* *}=x_{*}$ follows from the invertability of linear operator $M$ and the identity $M\left(x_{*}-x^{* *}\right)=F\left(x_{*}\right)-F\left(x^{* *}\right)=0-0=0$. 


\section{Local Convergence} by

Let $\ell, \ell_{j}, j=0,1,2,3,4$ be positive parameters. Define function $\psi_{1}:\left[0, \frac{1}{\ell_{0}}\right) \longrightarrow[0,+\infty)$

$$
\psi_{1}(t)=\frac{\ell t}{2\left(1-\ell_{0} t\right)}
$$

and set

$$
\rho_{A}=\frac{2}{2 \ell_{0}+\ell} .
$$

Define functions $q:\left[0, \frac{1}{\ell_{0}}\right) \longrightarrow[0,+\infty)$ by

$$
q(t)=\ell_{4}\left(1+\psi_{1}(t)\right) t-1 .
$$

By this definition, we have $q(0)=-1$ and $q(t) \longrightarrow+\infty$ as $t \longrightarrow{\frac{1}{\ell_{0}}}^{-}$. It then follows from the intermediate value theorem that function $q$ has zeros in $\left(0, \frac{1}{\ell_{0}}\right)$. Denote by $\rho_{q}$ the smallest such zero. Similarly, denote by $\rho_{p}$ the smallest zero of function $p:\left[0, \frac{1}{\ell_{0}}\right) \longrightarrow$ $[0,+\infty)$ defined by $p(t)=\ell_{0} \psi_{1}(t) t-1$. Set $\bar{\rho}=\min \left\{\rho_{q}, \rho_{p}\right\}$. Moreover, define function $\psi_{2}:[0, \bar{\rho}) \longrightarrow[0,+\infty)$ by

$$
\begin{aligned}
\psi_{2}(t)= & {\left[\frac{\ell \psi_{1}(t) t}{2\left(1-\ell_{0} \psi_{1}(t) t\right)}\right.} \\
& +\frac{\ell_{3} \ell_{4}\left(1+\psi_{1}(t) t\right) \psi_{1}(t) t}{\left(1-\ell_{0} \psi_{1}(t) t\right)\left(1-\ell_{1}\left(1+\psi_{1}(t)\right) t\right)} \\
& \left.+\frac{\ell_{2} \ell_{4}\left(1+\psi_{1}(t)\right)}{\left.\left(1-\ell_{1}\left(1+\psi_{1}(t)\right) t\right)\right)^{2}}\right] \psi_{1}(t) t .
\end{aligned}
$$

Set

$$
\mu(t)=\psi_{2}(t)-1 \text {. }
$$

We have again $\mu(0)=-1$ and $\mu(t) \longrightarrow+\infty$ as $t \longrightarrow \bar{\rho}^{-}$. Denote by $\rho_{\mu}$ the smallest zero of function $\mu$ in $(0, \bar{\rho})$. We shall show that

$$
\rho_{*}=\min \left\{\rho_{A}, \rho_{\mu}\right\}
$$

is a convergence radius for method (2). Set $T=\left[0, \rho_{*}\right)$. Then, it follows from these definitions that for each $t \in T$

$$
\begin{aligned}
& 0 \leq \ell_{0} t<1, \\
& 0 \leq q(t)<1, \\
& 0 \leq p(t)<1,
\end{aligned}
$$

and

$$
0 \leq \psi_{i}(t)<1, i=1,2 .
$$

The conditions $(C)$ shall be used together with the preceding notation provided that $x_{*}$ is a simple solution of equation $F(x)=0$.

Suppose:

(C1) For each $u \in D$

$$
\left\|F^{\prime}\left(x_{*}\right)^{-1}\left(F^{\prime}(u)-F^{\prime}\left(x_{*}\right)\right)\right\| \leq \ell_{0}\left\|u-x_{0}\right\| .
$$

Set $D_{2}=U\left(x_{*}, \frac{1}{\ell_{0}}\right) \cap D$.

(C2) For each $v, w \in D_{2}$

$$
\begin{gathered}
\left\|F^{\prime}\left(x_{*}\right)^{-1}\left(F^{\prime}(w)-F^{\prime}(v)\right)\right\| \leq \ell\|w-v\|, \\
\left\|F^{\prime}\left(x_{*}\right)^{-1} F^{\prime}(v)\right\| \leq \ell_{1}\left\|v-x_{*}\right\|,
\end{gathered}
$$




$$
\begin{gathered}
\left\|F^{\prime}\left(x_{*}\right)^{-1}\left([w, v ; F]-F^{\prime}(v)\right)\right\| \leq \ell_{2}(\|w-v\|), \\
\left\|F^{\prime}\left(x_{*}\right)^{-1}\left([w, v ; F]-F^{\prime}(w)\right)\right\| \leq \ell_{3}\|w-v\|
\end{gathered}
$$

and

$$
\left\|F^{\prime}\left(x_{*}\right)^{-1}\left([w, v ; F]-F^{\prime}\left(x_{*}\right)\right)\right\| \leq \ell_{4}\left(\left\|w-x_{*}\right\|+\left\|v-x_{*}\right\|\right) .
$$

(C3) $U\left[x_{*}, \rho_{*}\right] \subset D$.

Next, we present the local convergence analysis of method (2).

Theorem 2. Under the conditions (C) further suppose that $x_{0} \in U\left(x_{*}, \rho_{*}\right)-\left\{x_{*}\right\}$. Then, we have $\lim _{k \longrightarrow+\infty} x_{k}=x_{*}$.

Proof. We shall use mathematical induction to show

$$
\left\|y_{n}-x_{*}\right\| \leq \psi_{1}\left(\left\|x_{n}-x_{*}\right\|\right)\left\|x_{n}-x_{*}\right\| \leq\left\|x_{n}-x_{*}\right\|<\rho_{*}
$$

and

$$
\left\|x_{n+1}-x_{*}\right\| \leq \psi_{2}\left(\left\|x_{n}-x_{*}\right\|\right)\left\|x_{n}-x_{*}\right\| \leq\left\|x_{n}-x_{*}\right\| .
$$

where functions $\psi_{i}$ are given previously and radius $\rho_{*}$ is defined by (45). Let $z \in U\left(x_{*}, \rho_{*}\right)-$ $\left\{x_{*}\right\}$. Then, using (C1), (45) and (46), we obtain

$$
\left\|F^{\prime}\left(x_{*}\right)^{-1}\left(F^{\prime}(z)-F^{\prime}\left(x_{*}\right)\right)\right\| \leq \ell_{0}\left\|z-x_{*}\right\| \leq \ell_{0} \rho_{*}<1,
$$

so $F^{\prime}(z)$ is invertible with

$$
\left\|F^{\prime}(z)^{-1} F^{\prime}\left(x_{*}\right)\right\| \leq \frac{1}{1-\ell_{0}\left\|z-x_{*}\right\|^{\prime}}
$$

and iterate $y_{0}$ exists by (52) for $z=x_{0}$. Then, we can write

$$
y_{0}-x_{*}=\int_{0}^{1} F^{\prime}\left(x_{0}\right)^{-1}\left(F^{\prime}\left(x_{*}+\theta\left(x_{0}-x_{*}\right)\right)-F^{\prime}\left(x_{0}\right)\right) d \theta\left(x_{0}-x_{*}\right),
$$

so by (C1), (C2) and (52) (for $\left.z=x_{0}\right)$, we get

$$
\begin{aligned}
\left\|y_{0}-x_{*}\right\| & \leq \frac{\ell\left\|x_{0}-x_{*}\right\|^{2}}{2\left(1-\ell_{0}\left\|x_{0}-x_{*}\right\|\right)} \\
& \leq \psi_{1}\left(\left\|x_{0}-x_{*}\right\|\right)\left\|x_{0}-x_{*}\right\| \\
& \leq\left\|x_{0}-x_{*}\right\|<\rho_{*},
\end{aligned}
$$

so $y_{0} \in U\left(x_{*}, r\right)$ and (50) hold for $n=0$. As in (52), we also show

$$
\left\|F^{\prime}\left(y_{0}\right)^{-1} F^{\prime}\left(x_{*}\right)\right\| \leq \frac{1}{1-\ell_{0}\left\|y_{0}-x_{*}\right\|}
$$

and

$$
\left\|\left[y_{0}, x_{0} ; F\right]^{-1} F^{\prime}\left(x_{*}\right)\right\| \leq \frac{1}{1-\ell_{4}\left(\left\|y_{0}-x_{*}\right\|+\left\|x_{0}-x_{*}\right\|\right)},
$$


so iterate $x_{1}$ exists. Then, we can write in turn by the second substep of method (2) that

$$
\begin{aligned}
x_{1}-x_{*}= & y_{0}-x_{*}-F^{\prime}\left(y_{0}\right)^{-1} F\left(y_{0}\right) \\
& +\left(F^{\prime}\left(y_{0}\right)^{-1}-\left[y_{0}, x_{0} ; F\right]^{-1}\right) F\left(y_{0}\right) \\
& +\left(\left[y_{0}, x_{0} ; F\right]^{-1}-F^{\prime}\left(x_{0}\right)^{-1}\right) F\left(y_{0}\right) \\
= & y_{0}-x_{*}-F^{\prime}\left(y_{0}\right)^{-1} F\left(y_{0}\right) \\
& +F^{\prime}\left(y_{0}\right)^{-1}\left(\left[y_{0}, x_{0} ; F\right]-F^{\prime}\left(y_{0}\right)\right)\left[y_{0}, x_{0} ; F\right]^{-1} F\left(y_{0}\right) \\
& +\left[y_{0}, x_{0} ; F\right]^{-1}\left(F^{\prime}\left(x_{0}\right)-\left[y_{0}, x_{0} ; F\right]\right) F^{\prime}\left(x_{0}\right)^{-1} F\left(y_{0}\right) .
\end{aligned}
$$

Then, in view of (45), (49) (C2), (52) (for $\left.z=y_{0}\right)$ and (54)-(56), we get in turn that

$$
\begin{aligned}
\left\|x_{1}-x_{*}\right\| \leq & \frac{\ell\left\|y_{0}-x_{*}\right\|^{2}}{2\left(1-\ell_{0}\left\|y_{0}-x_{*}\right\|\right)} \\
& +\frac{\ell_{3}\left\|y_{0}-x_{0}\right\| \ell_{4}\left\|y_{0}-x_{*}\right\|}{\left(1-\ell_{0}\left\|y_{0}-x_{*}\right\|\right)\left(1-\ell_{1}\left(\left\|y_{0}-x_{*}\right\|+\left\|x_{0}-x_{*}\right\|\right)\right)} \\
& +\frac{\ell_{2}\left\|y_{0}-x_{0}\right\| \ell_{4}\left\|y_{0}-x_{*}\right\|}{\left(1-\ell_{1}\left(\left\|y_{0}-x_{*}\right\|+\left\|x_{0}-x_{*}\right\|\right)\right)^{2}} \\
\leq & \psi_{2}\left(\left\|x_{0}-x_{*}\right\|\right)\left\|x_{0}-x_{*}\right\| \leq\left\|x_{0}-x_{*}\right\|,
\end{aligned}
$$

showing (51) for $n=0$ and $x_{1} \in U\left(x_{*}, \rho_{*}\right)$, where we also used (53) and

$$
\begin{aligned}
\left\|y_{0}-x_{0}\right\| & \leq\left\|y_{0}-x_{*}\right\|+\left\|x_{*}-x_{0}\right\| \\
& \leq \psi_{1}\left(\left\|x_{0}-x_{*}\right\|\right)\left\|x_{0}-x_{*}\right\|+\left\|x_{0}-x_{*}\right\| \\
& =\left(1+\psi_{1}\left(\left\|x_{0}-x_{*}\right\|\right)\right)\left\|x_{0}-x_{*}\right\| .
\end{aligned}
$$

If we exchange $x_{0}, y_{0}, x_{1}$ by $x_{m}, y_{m}, x_{m+1}$, respectively, in the previous calculations we complete the induction for (50) and (51). Then, from the estimate

$$
\left\|x_{m+1}-x_{*}\right\| \leq \lambda_{1}\left\|x_{m}-x_{*}\right\|,
$$

where $\lambda_{1}=\psi_{2}\left(\left\|x_{0}-x_{*}\right\|\right) \in[0,1)$, we conclude $\lim _{m \longrightarrow+\infty} x_{m}=x_{*}$. We also have

$$
\left\|y_{m}-x_{*}\right\| \leq \lambda_{2}\left\|x_{m}-x_{*}\right\|<\rho_{*}
$$

where $\lambda_{2}=\psi_{1}\left(\left\|x_{0}-x_{*}\right\|\right) \in[0,1), \operatorname{solim}_{m \longrightarrow+\infty} y_{m}=x_{*}$.

Next, we present a uniqueness of the solution result.

Proposition 2. Suppose:

(a) $x_{*} \in D$ is a simple solution of equation $F(x)=0$.

(b) There exists $\tilde{s} \geq 0$ such that

$$
\frac{\ell_{0}}{2} \tilde{s}<1 .
$$

Set $D_{4}=D \cap U\left[x_{*}, \tilde{s}\right]$. Then, the only solution of equation $F(x)=0$ in the region $D_{4}$ is $x_{*}$.

Proof. Let $x^{* *} \in D_{4}$ with $F\left(x^{* *}\right)=0$. Set $Q=\int_{0}^{1} F^{\prime}\left(x_{*}+\theta\left(x^{* *}-x_{*}\right)\right) d \theta$. Then, using (C1) and (60), we obtain

$$
\begin{aligned}
\left\|F^{\prime}\left(x_{*}\right)^{-1}\left(Q-F^{\prime}\left(x_{*}\right)\right)\right\| & \leq \frac{\ell_{0}}{2}\left\|x^{* *}-x_{*}\right\| \\
& \leq \frac{\ell_{0}}{2} \tilde{s}<1,
\end{aligned}
$$

so $x^{* *}=x_{*}$, since $Q^{-1} \in L\left(E_{1}, E\right)$ and $Q\left(x^{* *}-x_{*}\right)=F\left(x^{* *}\right)-F\left(x_{*}\right)=0-0=0$. 


\section{Numerical Experiments}

We provide some examples, showing that the old convergence criteria are not verified, but ours are. The divided difference is chosen by

$$
[u, v ; F]=\int_{0}^{1} F^{\prime}(v+\theta(u-v)) d \theta .
$$

Example 1. Define function

$$
h(t)=c_{0} t+c_{1}+c_{2} \sin c_{3} t, t_{0}=0,
$$

where $c_{j}, j=0,1,2,3$ are parameters. Then, clearly for $c_{3}$ large and $c_{2}$ small, $\frac{L_{0}}{L}$ can be small (arbitrarily).

Example 2. Let $E=E_{1}=H([0,1])$ the domain of functions given on $[0,1]$, which are continuous. We consider the max-norm. Choose $D=U(0, d), d>1$. Define $G$ on $D$ be

$$
G(x)(s)=x(s)-w(s)-\epsilon \int_{0}^{1} P(s, t) x^{3}(t) d t,
$$

$x \in E, s \in[0,1], w \in E$ is given, $\epsilon$ is a parameter and $P$ is the Green's kernel given by

$$
P\left(\epsilon_{2}, \epsilon_{1}\right)= \begin{cases}\left(1-\epsilon_{2}\right) \epsilon_{1}, & \epsilon_{1} \leq \epsilon_{2} \\ \epsilon_{2}\left(1-\epsilon_{1}\right), & \epsilon_{2} \leq \epsilon_{1}\end{cases}
$$

By (61), we have

$$
\left(G^{\prime}(x)(z)\right)(s)=z(s)-3 \epsilon \int_{0}^{1} P(s, t) x^{2}(t) z(t) d t,
$$

$t \in E, s \in[0,1]$. Consider $x_{0}(s)=w(s)=1$ and $|\epsilon|<\frac{8}{3}$. We get

$$
\begin{gathered}
\left\|I-G^{\prime}\left(x_{0}\right)\right\|<\frac{3}{8}|\epsilon|, G^{\prime}\left(x_{0}\right)^{-1} \in L\left(E_{1}, E\right), \\
\left\|F^{\prime}\left(x_{0}\right)^{-1}\right\| \leq \frac{8}{8-3|\epsilon|}, \eta=\frac{|\epsilon|}{8-3|\epsilon|}, L_{0}=\frac{12|\epsilon|}{8-3|\epsilon|},
\end{gathered}
$$

and $L=\frac{6 \eta|\epsilon|}{8-3|\epsilon|}$

Example 3. Let $E, E_{1}$ and $D$ be as in the Example 5.3. It is well known that the boundary value problem [4]

$$
\begin{gathered}
\xi(0)=0,(1)=1, \\
\xi^{\prime \prime}=-\xi-\lambda \xi^{2}
\end{gathered}
$$

can be given as a Hammerstein-like nonlinear integral equation

$$
\xi(s)=s+\int_{0}^{1} K(s, t)\left(\xi^{3}(t)+\lambda \xi^{2}(t)\right) d t
$$

where $\lambda$ is a parameter. Then, define $F: D \longrightarrow E_{1}$ by

$$
[F(x)](s)=x(s)-s-\int_{0}^{1} K(s, t)\left(x^{3}(t)+\lambda x^{2}(t)\right) d t .
$$


Choose $\xi_{0}(s)=s$ and $D=U\left(\xi_{0}, \rho_{0}\right)$. Then, clearly $U\left(\xi_{0}, \rho_{0}\right) \subset U\left(0, \rho_{0}+1\right)$, since $\left\|\xi_{0}\right\|=1$. Suppose $2 \lambda<5$. Then, conditions $(A)$ are satisfied for

$$
L_{0}=\frac{2 \lambda+3 \rho_{0}+6}{8}, L=\frac{\lambda+6 \rho_{0}+3}{4}
$$

and $\eta=\frac{1+\lambda}{5-2 \lambda}$. Notice that $L_{0}<L$.

Example 4. Consider the motion system

$$
T_{1}^{\prime}(x)=e^{x}, T_{2}^{\prime}(y)=(e-1) y+1, T_{3}^{\prime}(z)=1
$$

with $T_{1}(0)=T_{2}(0)=T_{3}(0)=0$. Let $T=\left(T_{1}, T_{2}, T_{3}\right)$. Let $E=E_{1}=\mathbb{R}^{3}, D=B[0,1], x_{*}=$ $(0,0,0)^{T}$. Define function $T$ on $D$ for $w=(x, y, z)^{T}$ by

$$
T(w)=\left(e^{x}-1, \frac{e-1}{2} y^{2}+y, z\right)^{T} .
$$

Then, we get

$$
T^{\prime}(v)=\left[\begin{array}{ccc}
e^{x} & 0 & 0 \\
0 & (e-1) y+1 & 0 \\
0 & 0 & 1
\end{array}\right],
$$

so $\ell_{0}=e-1, \ell=e^{\frac{1}{e-1}}=\ell_{1}, \ell_{2}=\ell_{3}=\frac{\ell}{2}, \ell_{4}=\frac{\ell_{0}}{2}$. Then, the radii are:

$$
\rho_{A}=0.3827=\rho^{*}, \rho_{\mu}=1.7156 .
$$

\section{Conclusions}

A finer convergence analysis is presented for method (2) utilizing generalized conditions. This analysis includes weaker criteria of convergence and computable error bounds not given in earlier papers.

Author Contributions: Conceptualization, C.I.A., I.K.A., J.J., S.R. and S.G.; methodology, C.I.A., I.K.A., J.J., S.R. and S.G.; software, C.I.A., I.K.A., J.J., S.R. and S.G.; validation, C.I.A., I.K.A., J.J., S.R. and S.G.; formal analysis, C.I.A., I.K.A., J.J., S.R. and S.G.; investigation, C.I.A., I.K.A., J.J., S.R. and S.G.; resources, C.I.A., I.K.A., J.J., S.R. and S.G.; data curation, C.I.A., I.K.A., J.J., S.R. and S.G.; writing—original draft preparation, C.I.A., I.K.A., J.J., S.R. and S.G.; writing-review and editing, C.I.A., I.K.A., J.J., S.R. and S.G.; visualization, C.I.A., I.K.A., J.J., S.R. and S.G.; supervision, C.I.A., I.K.A., J.J., S.R. and S.G.; project administration, C.I.A., I.K.A., J.J., S.R. and S.G.; funding acquisition, C.I.A., I.K.A., J.J., S.R. and S.G. All authors have read and agreed to the published version of the manuscript.

Funding: This research received no external funding.

Institutional Review Board Statement: Not applicable.

Informed Consent Statement: Not applicable.

Conflicts of Interest: The authors declare no conflict of interest.

\section{References}

1. Chen, X.; Yamamoto, T.; Convergence domains of certain iterative methods for solving nonlinear equations. Numer. Funct. Anal. Optim. 1989, 10, 37-48. [CrossRef]

2. Đukić, D.; Paunović, L.; Radenović, S. Convergence of iterates with errors of uniformly quasi-Lipschitzian mappings in cone metric spaces. Kragujev. J. Math. 2011, 35, 399-410.

3. Ezquerro, J.A.; Gutiérrez, J.M.; Hernández, M.A.; Romero, N.; Rubio, M.J. The Newton method: From Newton to Kantorovich (Spanish). Gac. R. Soc. Mat. Esp. 2010, 13, 53-76.

4. Ezquerro, J.A.; Hernandez, M.A. Newton's Method: An Updated Approach of Kantorovich's Theory; Birkhäuser: Cham, Switzerland, 2018.

5. Grau-Sánchez, M.; Àngela, G.; Noguera, M. Ostrowski type methods for solving systems of nonlinear equations. Appl. Math. Comput. 2011, 218, 2377-2385. [CrossRef] 
6. Magréñan,A. A.; Gutiérrez, J.M. Real dynamics for damped Newton's method applied to cubic polynomials. J. Comput. Appl. Math. 2015, 275, 527-538. [CrossRef]

7. Nashed, M.Z.; Chen, X. Convergence of Newton-like methods for singular operator equations using outer inverses. Numer. Math. 1993, 66, 235-257. [CrossRef]

8. Proinov P.D. New general convergence theory for iterative processes and its applications to Newton-Kantorovich type theorems. J. Complex. 2010, 26, 3-42. [CrossRef]

9. Todorcević, V. Harmonic Quasiconformal Mappings and Hyperbolic Type Metrics; Springer Nature AG: Cham, Switzerland, 2019.

10. Yamamoto, T. A convergence theorem for Newton-like methods in Banach spaces. Numer. Math. 1987, 51, 545-557. [CrossRef]

11. Argyros, I.K. On the Newton-Kantorovich hypothesis for solving equations. J. Comput. Math. 2004, 169, 315-332. [CrossRef]

12. Argyros, I.K. Computational Theory of Iterative Methods; Series: Studies in Computational Mathematics; Chui, C.K., Wuytack, L., Eds.; Elsevier Publ. Co.: New York, NY, USA, 2007; Volume 15.

13. Argyros, I.K. Convergence and Applications of Newton-Type Iterations; Springer: Berlin, Germany, 2008.

14. Argyros, I.K.; Hilout, S. Weaker conditions for the convergence of Newton's method. J. Complex. 2012, 28, 364-387. [CrossRef]

15. Argyros, I.K.; Hilout, S. On an improved convergence analysis of Newton's method. Appl. Math. Comput. 2013, 225, 372-386. [CrossRef]

16. Argyros, I.K.; Magréñan, A.A. Iterative Methods and Their Dynamics with Applications; CRC Press: New York, NY, USA, 2017.

17. Argyros, I. K.; Magréñan, A.A. A Contemporary Study of Iterative Methods; Elsevier (Academic Press): New York, NY, USA, 2018.

18. Behl, R.; Maroju, P.; Martinez, E.; Singh, S. A study of the local convergence of a fifth order iterative method. Indian J. Pure Appl. Math. 2020, 51, 439-455.

19. Cătinaş, Emil The inexact, inexact perturbed, and quasi-Newton methods are equivalent models. Math. Comp. 2005, 74, 291-301. [CrossRef]

20. Dennis, J.E., Jr. On Newton-like methods. Numer. Math. 1968, 11, 324-330. [CrossRef]

21. Dennis, J.E., Jr.; Schnabel, R.B. Numerical Methods for Unconstrained Optimization and Nonlinear Equations; SIAM: Philadelphia, PA, USA, 1996.

22. Deuflhard, P.; Heindl, G. Affine invariant convergence theorems for Newton's method and extensions to related methods. SIAM J. Numer. Anal. 1979, 16, 1-10. [CrossRef]

23. Deuflhard, P. Newton Methods for Nonlinear Problems. Affine Invariance and Adaptive Algorithms; Springer Series in Computational Mathematics; Springer: Berlin, Germany, 2004; Volume 35.

24. Gutiérrez, J.M.; Magreñán, Á.A.; Romero, N. On the semilocal convergence of Newton-Kantorovich method under centerLipschitz conditions. Appl. Math. Comput. 2013, 221, 79-88. [CrossRef]

25. Hernandez, M.A.; Romero, N. On a characterization of some Newton-like methods of R-order at least three. J. Comput. Appl. Math. 2005, 183, 53-66. [CrossRef]

26. Magréñan, A.A.; Argyros, I.K.; Rainer, J.J.; Sicilia, J.A. Ball convergence of a sixth-order Newton-like method based on means under weak conditions. J. Math. Chem. 2018, 56, 2117-2131. [CrossRef]

27. Ortega, J.M.; ; Rheinboldt, W.C. Iterative Solution of Nonlinear Equations in Several Variables; SIAM Publ.: Philadelphia, PA, USA, 2000.

28. Proinov P.D. General local convergence theory for a class of iterative processes and its applications to Newton's method. J. Complex. 2009, 25, 38-62. [CrossRef]

29. Rheinboldt, W.C. An Adaptive Continuation Process of Solving Systems of Nonlinear Equations; Polish Academy of Science, Banach Center Publisher: Warsaw, Poland, 1978; Volume 3, pp. 129-142.

30. Shakhno, S.M.; Gnatyshyn, O.P. On aan iterative algorithm of order 1.839... for solving nonlinear least squares problems. Appl. Math. Appl. 2005, 161, 253-264.

31. Shakhno, S.M.; Iakymchuk, R.P.; Yarmola, H.P. Convergence analysis of a two step method for the nonlinear squares problem with decomposition of operator. J. Numer. Appl. Math. 2018, 128, 82-95.

32. Sharma, J.R.; Guha, R.K.; Sharma, R. An efficient fourth order weighted-Newton method for systems of nonlinear equations. Numer. Algorithms 2013, 62, 307-323. [CrossRef]

33. Soleymani, F.; Lotfi, T.; Bakhtiari, P. A multi-step class of iterative methods for nonlinear systems. Optim. Lett. 2014, 8, 1001-1015. [CrossRef]

34. Steffensen, J.F. Remarks on iteration. Skand Aktuar Tidsr. 1993, 16, 64-72. [CrossRef]

35. Traub, J.F. Iterative Methods for the Solution of Equations; Prentice Hall: Hoboken, NJ, USA, 1964.

36. Traub, J.F.; Werschulz, A.G. Complexity and Information; Lincei Lectures; Cambridge University Press: Cambridge, UK, 1998; xii+139pp.; ISBN 0-521-48506-1.

37. Traub, J.F.; Wozniakowski, H. Path integration on a quantum computer. Quantum Inf. Process 2002, 1, 356-388. [CrossRef]

38. Verma, R. New Trends in Fractional Programming; Nova Science Publisher: New York, NY, USA, 2019.

39. Zabrejko, P.P.; Nguen, D.F. The majorant method in the theory of Newton-Kantorovich approximations and the Pták error estimates. Numer. Funct. Anal. Optim. 1987, 9, 671-684. [CrossRef]

40. Potra, F.A.; Pták, V. Nondiscrete Induction and Iterative Processes; Research Notes in Mathematics; Pitman (Advanced Publishing Program): Boston, MA, USA, 1984; Volume 103.

41. Kantorovich, L.V.; Akilov, G.P. Functional Analysis; Pergamon Press: Oxford, UK, 1982.

42. Ortega, J.M.; Rheinboldt, W.C. Iterative Solution of Nonlinear Equations in Several Variables; Academic Press: New York, NY, USA, 1970. 